\title{
Increased prevalence of proliferative retinopathy in patients with type 1 diabetes who are deficient in glucose-6-phosphate dehydrogenase
}

\author{
G. Cappai • M. Songini • A. Doria • J. D. Cavallerano • \\ M. Lorenzi
}

Received: 8 November 2010 / Accepted: 4 February 2011 /Published online: 5 March 2011

(C) Springer-Verlag 2011

\begin{abstract}
Aims/hypothesis Impaired activity of the pentose phosphate pathway of glucose metabolism caused by hereditary deficiency of its key regulatory enzyme glucose-6phosphate dehydrogenase (G6PD) has consequences that may worsen or attenuate the course of diabetic complications. Decreased availability of NADPH can predispose to oxidative stress and endothelial dysfunction, but can also limit the activity of the polyol pathway and cholesterol synthesis. Reduced availability of pentose phosphates for nucleic acid synthesis could impair cell proliferation. We sought to learn in which direction G6PD deficiency affects diabetic retinopathy.

Methods We enrolled patients who were G6PD-deficient or -sufficient with type 1 diabetes of duration 15 years or
\end{abstract}

G. Cappai

Ophthalmology Clinic, Ospedale San Giovanni di Dio,

Cagliari, Italy

M. Songini

Diabetes Center, Ospedale San Michele,

Cagliari, Italy

A. Doria

Joslin Diabetes Center, Harvard Medical School,

Boston, MA, USA

\section{J. D. Cavallerano}

Beetham Eye Institute at Joslin Diabetes Center,

Harvard Medical School,

Boston, MA, USA

\section{Lorenzi ( $\square)$}

Schepens Eye Research Institute, Harvard Medical School,

20 Staniford Street,

Boston, MA 02114, USA

e-mail: mara.lorenzi@schepens.harvard.edu longer for whom $\mathrm{HbA}_{1 \mathrm{c}}$ records were available for at least the previous 3 years. Renal failure and smoking were exclusion criteria. For each participant seven standard field colour photographs were obtained of each eye, and retinopathy was graded in a masked fashion.

Results The clinical characteristics of the 19 G6PDdeficient patients studied (age $42 \pm 9$ years, diabetes duration $24 \pm 6$ years, average $\mathrm{HbA}_{1 \mathrm{c}}$ over 3 years $6.7 \pm$ $0.8 \%$ ) were similar to those of the 35 G6PD-sufficient patients. Almost $90 \%$ of patients in both groups had retinopathy; however, proliferative retinopathy was noted solely among G6PD-deficient patients $(28 \%, p=$ 0.0036 vs G6PD-sufficient). The G6PD-deficient patients also showed a trend for increased frequency of microalbuminuria.

Conclusions/interpretation The data suggest that G6PD deficiency accelerates the microvascular complications of diabetes, and that among the consequences of G6PD deficiency those that can enhance the damage caused by diabetes outweigh those that could be protective.

Keywords Diabetic retinopathy - Endothelial nitric oxide synthase - G6PD deficiency · Oxidative stress · Polyol pathway Proliferative retinopathy Type 1 diabetes
Abbreviations
EDIC Epidemiology of Diabetes Interventions and Complications
eNOS Endothelial nitric oxide synthase
ETDRS Early Treatment Diabetic Retinopathy Study
G6PD Glucose-6-phosphate dehydrogenase
NPDR Non-proliferative diabetic retinopathy
PDR Proliferative diabetic retinopathy 


\section{Introduction}

Diabetic retinopathy remains a complication of diabetes even among individuals who have made a systematic and long-term attempt to optimise glycaemic control [1]. New approaches to lessen its development or progression are desirable. Genetic variations offer the opportunity to identify, in humans, factors that modulate the pathogenic process and may suggest targets for adjunct therapy.

Glucose-6-phosphate dehydrogenase (G6PD) is the key regulatory enzyme of the pentose phosphate pathway of glucose metabolism, which provides pentose phosphates for nucleic acid synthesis and NADPH for synthetic reactions and maintenance of the cellular redox status. G6PD deficiency is the most common enzymopathy in humans. It is a hereditary abnormality in which the activity or stability of the enzyme is greatly diminished. As the gene determining the structure of G6PD is on the X chromosome, inheritance is sex-linked with men expressing the full defect.

The consequences of G6PD deficiency could worsen, but also attenuate, the processes leading to diabetic microangiopathy. On the one hand, NADPH is required to regenerate reduced glutathione, and thus G6PD deficiency facilitates oxidative stress. The main clinical manifestation of G6PD deficiency is a haemolytic anaemia, occurring because the erythrocytes, which rely exclusively on the pentose phosphate pathway to generate NADPH, are insufficiently protected from oxidation in the presence of drugs, infections and consumption of broad beans (or fava beans, 'favism') [2]. G6PD deficiency could also contribute to diabetic vascular disease by magnifying endothelial dysfunction, as NADPH is a required cofactor for endothelial nitric oxide synthase (eNOS) [3]. On the other hand, the reduced cellular supply of NADPH could protect from mechanisms of diabetic tissue damage. By dampening the activity of aldose reductase, which preferentially uses NADPH as a cofactor to convert glucose to sorbitol, the limited NADPH supply could prevent polyol pathway activity. Decreased activity of the first step of the pathway has been observed in cultured fibroblasts from individuals with G6PD deficiency [4], supporting the concepts that the NADPH supply is decreased not only in erythrocytes but also in nucleated cells [5], and that the decreased supply may thus limit the contribution of excess polyol pathway activity to retinopathy and other complications of diabetes. G6PD deficiency could attenuate diabetic vascular disease also on account of decreased cholesterol synthesis and a favourable lipid profile [5], as NADPH is a cofactor for 3hydroxy-3-methylglutaryl-CoA (HMG-CoA) reductase. In view of the multiplicity of reactions affected by the products of the pentose phosphate pathway, the net effect of G6PD deficiency on a specific tissue or function is difficult to predict. The findings on cardiovascular mortality of Sardinian G6PD-deficient men illustrate the challenge: expected to be increased on account of the widely hypothesised role of oxidative stress in atherothrombotic disorders, cardiovascular mortality was instead found to be substantially reduced [2].

To learn experimentally whether and in which direction G6PD deficiency affects diabetic retinopathy, we took advantage of the epidemiology of type 1 diabetes and G6PD deficiency in the Italian island of Sardinia. Sardinia has one of the highest incidences of type 1 diabetes in the world [6], second only to Finland, and a very high prevalence of G6PD deficiency (present in $12-15 \%$ of the male population compared with $0.48 \%$ in continental Italy) [2]. In Sardinia, all variants of G6PD deficiency are due to the same mutation, which results in only $5-10 \%$ residual activity of the enzyme [7] and G6PD deficiency does not show an association with diabetes [8]. We could thus plan to compare the prevalence and severity of retinopathy in diabetic patients with a homogeneously severe degree of G6PD deficiency and in patients with the same duration of type 1 diabetes and the same glycaemic control but with normal G6PD activity.

\section{Methods}

The institutional review boards of the Schepens Eye Research Institute and the Joslin Diabetes Center in Boston, USA, and of the Azienda Ospedaliera G. Brotzu in Cagliari, Italy, approved the study. The central recruitment site was the Diabetes Center of the Ospedale San Michele in Cagliari (Azienda Ospedaliera G. Brotzu); additional referrals were received from local diabetes specialists. We recruited male patients, 18-55 years of age with type 1 diabetes of duration 15 years or longer, with or without G6PD deficiency. An essential criterion for inclusion in the study was the availability, through databases, of $\mathrm{HbA}_{1 \mathrm{c}}$ records spanning at least 3 years. Exclusion criteria were retinal or systemic diseases other than diabetes, renal insufficiency (serum creatinine $\geq 132.6 \mu \mathrm{mol} / \mathrm{l}$ ) and smoking. After the participants had given informed consent, we retrieved the sequential measurements of $\mathrm{HbA}_{1 \mathrm{c}}$ over the preceding 3 years, as well as the measurements of serum creatinine, albumin excretion rate (overnight urine collections), and serum lipids obtained within the preceding 6 months. Erythrocyte G6PD activity was either available in the databases or measured for this study by the Clinical Laboratories of the San Michele Hospital. Blood pressure was measured at the enrolment visit.

Each participant underwent a complete eye examination. A photographer took Early Treatment Diabetic Retinopathy Study (ETDRS) seven standard field $35 \mathrm{~mm}$ stereoscopic 
colour photographs of each eye using a $35 \mathrm{~mm}$ colour slide film and a standard mydriatic fundus camera. An experienced grader (J. D. Cavallerano), masked to all patient characteristics, graded the slides according to modified ETDRS protocol [9]. The retinopathy grade assigned to the individual patient was that for the eye with the more advanced level of retinopathy. Macular oedema was an independent notation.

The variables in the two groups of patients were compared using a two-tailed $t$ test, except in the case of binary or categorical variables, for which the frequencies were compared by means of Fisher's exact tests.

\section{Results}

Table 1 presents the characteristics of the patients studied and the comparisons between type 1 diabetic patients with and without G6PD deficiency. Age and duration of diabetes were almost identical. The $\mathrm{HbA}_{1 \mathrm{c}}$ levels were, on average, $1 \%$ lower in the G6PD-deficient group ( $p=0.0001$ vs controls), consistent with the extent by which $\mathrm{HbA}_{1 \mathrm{c}}$ is lower in G6PD-deficient vs G6PD-sufficient non-diabetic individuals [10], most likely reflecting increased erythrocyte turnover. The prevalence of any retinopathy approached $90 \%$ in both groups; however, PDR was present only among patients with G6PD deficiency ( $p=$ 0.0036 vs the control group). Four of the five cases were quiescent PDR treated with laser, and one was PDR without high-risk characteristics. Consistent with the increased prevalence of PDR, there was a tendency for an increased frequency of retinopathy grade 4 or higher in the G6PDdeficient group compared with the control group (39\% vs $18 \% ; p=0.17)$. The G6PD-deficient group also showed a trend for increased prevalence of microalbuminuria, although the $p$ value did not reach significance (Table 1).

\section{Discussion}

By having no cases of PDR among 33 patients with $24 \pm$ 7 years of diabetes, the population of type 1 diabetic patients with normal G6PD examined in this study compares very favourably with larger series of intensively treated patients. The prevalence of PDR in the DCCTEpidemiology of Diabetes Interventions and Complications (EDIC) patients with a duration of diabetes $(25 \pm 5$ years $)$ and $\mathrm{HbA}_{1 \mathrm{c}}$ levels similar to those of our patients was $4.4 \%$ in intensively treated patients from the prevention arm of the DCCT, and $13.2 \%$ in patients from the intervention arm [1]. In our G6PD-deficient patients with $24 \pm 6$ years of diabetes the frequency of PDR $(28 \%)$ was much higher than in the control group and the DCCT-EDIC cohorts; the
Table 1 Characteristics of the diabetic patients studied and prevalence of complications

\begin{tabular}{|c|c|c|c|}
\hline Characteristic & $\begin{array}{l}\text { Type } 1 \\
\text { diabetes } \\
n=35\end{array}$ & $\begin{array}{l}\text { Type } 1 \text { diabetes, } \\
\text { G6PD-deficient } \\
n=19\end{array}$ & $p$ value \\
\hline Age (years) & $42.6 \pm 8.5$ & $42.0 \pm 8.7$ & 0.8 \\
\hline Male sex $(\%)$ & 100 & 100 & \\
\hline Diabetes duration (years) & $24.0 \pm 7.2$ & $24.4 \pm 6.0$ & 0.8 \\
\hline G6PD activity $(\mathrm{U} / \mathrm{g} \mathrm{Hb})^{\mathrm{a}}$ & $13.5 \pm 3.2$ & $2.0 \pm 0.6$ & $<0.0001$ \\
\hline $\mathrm{HbA}_{1 \mathrm{c}}(\%)^{\mathrm{b}}$ & $7.8 \pm 0.9$ & $6.7 \pm 0.8$ & 0.0001 \\
\hline $\mathrm{HbA}_{1 \mathrm{c}}$ measurements $(n)^{\mathrm{c}}$ & $8.8 \pm 2.3$ & $9.2 \pm 2.1$ & 0.5 \\
\hline Blood pressure, systolic (mmHg) & $122 \pm 10$ & $126 \pm 10$ & 0.15 \\
\hline Blood pressure, diastolic $(\mathrm{mmHg})$ & $74 \pm 7$ & $77 \pm 5$ & 0.09 \\
\hline Albumin excretion rate $(\mu \mathrm{g} / \mathrm{min})^{\mathrm{d}}$ & $8(5-16)$ & $16(7-40)$ & 0.14 \\
\hline Microalbuminuria, $n(\%)^{\mathrm{e}}$ & $6(17.4)$ & $7(36.8)$ & 0.18 \\
\hline Serum creatinine $(\mu \mathrm{mol} / \mathrm{l})$ & $79.6 \pm 8.8$ & $79.6 \pm 8.8$ & 0.9 \\
\hline Total cholesterol (mmol/l) & $4.7 \pm 0.8$ & $5.2 \pm 1.3$ & 0.26 \\
\hline HDL-cholesterol (mmol/l) & $1.5 \pm 0.3$ & $1.7 \pm 0.6$ & 0.31 \\
\hline Intraocular pressure $\mathrm{R}(\mathrm{mmHg})$ & $14.5 \pm 1.9$ & $14.1 \pm 1.9$ & 0.43 \\
\hline Intraocular pressure $\mathrm{L}(\mathrm{mmHg})$ & $14.6 \pm 1.8$ & $13.9 \pm 2.0$ & 0.19 \\
\hline Retinopathy, any $n(\%)^{\mathrm{f}}$ & $29(88)$ & $16(89)$ & 0.99 \\
\hline Retinopathy, proliferativen (\%) & 0 & $5(28)$ & 0.0036 \\
\hline $\begin{array}{l}\text { Macular oedema in at least one } \\
\text { eye, } n(\%)^{\mathrm{g}}\end{array}$ & $12(36)$ & $5(28)$ & 0.76 \\
\hline
\end{tabular}

Data are means \pm SD or percentages, except as indicated

${ }^{\text {a }}$ Reflects measurements performed by the same laboratory (G6PD kit, bcs Biotech, Cagliari, Italy) in 29 of the diabetic patients with normal G6PD and ten of the G6PD-deficient diabetic patients. Values for G6PD activity in the remaining patients were obtained by diverse methods, and the values for the G6PD-deficient patients were indicated as $0 \mathrm{U} / \mathrm{g} \mathrm{Hb}$. These data were not used in computations

${ }^{\mathrm{b}}$ Reflects measurements recorded over the preceding 3 years

${ }^{c}$ Number of measurements available over the preceding 3 years for each patient

${ }^{\mathrm{d}}$ Presented as median and interquartile range. Comparison between the two groups is based on log-transformed values to approximate the normal distribution

${ }^{\mathrm{e}}$ Microalbuminuria was considered present when the albumin excretion rate was $20 \mu \mathrm{g} / \mathrm{min}$ or greater

${ }^{\mathrm{f}}$ Retinal photographs were available and graded in 33 of the diabetic patients with normal G6PD and 18 of the G6PD-deficient patients. Diabetic retinopathy was graded 0 if absent, 1 if questionable, 2 if mild NPDR, 3 if moderate NPDR, 4 if severe NPDR, 5 if very severe NPDR, 6 if PDR without high-risk characteristics, 7 if PDR with high-risk characteristics and 8 if quiescent PDR

${ }^{\mathrm{g}}$ The presence and degree of macular oedema was determined from review of the retinal photographs according to the ETDRS criteria. Macular oedema was defined as thickening of the retina within one disc diameter of the centre of the macula and/or hard exudates greater than or equal to standard photograph 3 in a standard 30-degree photographic field centred on the macula, with some hard exudates within one disc diameter of the centre of the macula

$\mathrm{L}$, left; R, right

frequency of severe and very severe NPDR and of microalbuminuria also tended to be greater than in the control group. Glycaemic exposure could not be assessed precisely because the relationship of glycaemic levels to $\mathrm{HbA}_{1 \mathrm{c}}$ is 
altered in G6PD-deficient patients [10], and longitudinal measurements of indices not based on erythrocytes were not available. However, we documented that the $\mathrm{HbA}_{1 \mathrm{c}}$ levels of our G6PD-deficient patients were lower than those of G6PD-sufficient patients, and by the extent attributed in non-diabetic individuals to the erythrocyte abnormalities [10]. Drugs that block the renin-angiotensin system and may introduce a confounder because they slow the progression of diabetic retinopathy [11] were taken by only two of the G6PD-sufficient and one of the G6PD-deficient patients.

These considerations suggest that G6PD deficiency accelerates the microvascular complications of diabetes and that, of the many consequences of G6PD deficiency, those that can enhance the damage caused by diabetes outweigh those that could be protective. The decreased supply of NADPH, by limiting the regeneration of reduced glutathione, may magnify any level of oxidant stress occurring in diabetes. Additionally, if the observations made in cultured endothelial cells and in animals [3] apply to human G6PD deficiency, the decreased NADPH supply could affect eNOS activity, and thus reduce NO availability and endothelial performance. Of note, a facet of the impaired endothelial performance in in vitro and in vivo models of G6PD deficiency is an attenuated response to vascular endothelial growth factor and decreased angiogenesis [3]. Our diabetic patients with G6PD deficiency showed that the endothelial cells of retinal vessels respond to angiogenic cues and form new vessels. A recent report on pterygium (a benign growth of conjunctival fibroblasts) documents that nucleated cells of G6PD-deficient individuals, when in need of NADPH and pentoses for the synthetic activities of rapid growth, can compensate for the instability of G6PD by increasing its production [7].

Although this was a small study that awaits confirmation, it suggests that diabetic patients with G6PD deficiency can benefit from an additional level of intensive treatment to lessen the risk or severity of complications. It also encourages the testing of hypotheses to explain how G6PD deficiency augments the effects of diabetes on the microvasculature. One testable hypothesis is that G6PD-deficient patients have decreased NO availability and endothelial dysfunction. Experimental data in favour or against this hypothesis could inform therapeutic approaches to diabetic retinopathy.

Acknowledgements We are grateful to M. Fossarello, Director of the Ophthalmology Clinic, Ospedale San Giovanni di Dio, University of Cagliari for making available for this study facilities and personnel;
S. Pisano of the Ophthalmology Clinic, Ospedale San Giovanni di Dio for taking the eye photographs; and F. Mastinu and M. Mastinu for referring patients. The study was supported by a grant from the Juvenile Diabetes Research Foundation, a grant-in-aid from the Massachusetts Lions Eye Research Fund, and a generous donation from B. Shaw to M. Lorenzi.

Duality of interest The authors declare that there is no duality of interest associated with this manuscript.

\section{References}

1. Nathan DM, Zinman B, Cleary PA, Diabetes Control and Complications Trial/Epidemiology of Diabetes Interventions and Complications (DCCT/EDIC) Research Group et al (2009) Modern-day clinical course of type 1 diabetes mellitus after 30 years' duration: the diabetes control and complications trial/ epidemiology of diabetes interventions and complications and Pittsburgh epidemiology of diabetes complications experience (1983-2005). Arch Intern Med 169:1307-1316

2. Cocco P, Todde P, Fornera S, Manca MB, Manca P, Sias AR (1998) Mortality in a cohort of men expressing the glucose-6phosphate dehydrogenase deficiency. Blood 91:706-709

3. Leopold JA, Walker J, Scribner AW et al (2003) Glucose-6phosphate dehydrogenase modulates vascular endothelial growth factor-mediated angiogenesis. J Biol Chem 278:32100 32106

4. Kennedy A, Frank RN, Varma SD (1983) Galactitol accumulation by glucose-6-phosphate deficient fibroblasts: a cellular model for resistance to the complications of diabetes mellitus. Life Sci 33:1277-1283

5. Muntoni S, Batetta B, Dessi S, Muntoni S, Pani P (1992) Serum lipoprotein profile in the Mediterranean variant of glucose-6phosphate dehydrogenase deficiency. Eur J Epidemiol 8(Suppl 1):48-53

6. Casu A, Pascutto C, Bernardinelli L, Songini M, the Sardinian IDDM Epidemiology Study Group (2004) Type 1 diabetes among Sardinian children is increasing: the Sardinian diabetes register for children aged 0-14 years (1989-1999). Diabetes Care 27:1623-1629

7. Peiretti E, Mandas A, Cocco P et al (2010) Glucose-6-phosphatedehydrogenase deficiency as a risk factor for pterygium. Invest Ophthalmol Vis Sci 51:2928-2935

8. Meloni T, Pacifico A, Forteleoni G, Meloni GF (1992) G6PD deficiency and diabetes mellitus in northern Sardinian subjects. Haematologica 77:94-95

9. Early Treatment Diabetic Retinopathy Study Research Group (1991) Grading diabetic retinopathy from stereoscopic color fundus photographs - an extension of the modified Airlie House Classification: ETDRS Report Number 10. Ophthalmology 98:786-806

10. Meloni T, Pacifico A, Forteleoni G, Meloni GF (1994) HbAlc levels in diabetic Sardinian patients with or without G6PD deficiency. Diabetes Res Clin Pract 23:59-61

11. Mauer M, Zinman B, Gardiner R et al (2009) Renal and retinal effects of enalapril and losartan in type 1 diabetes. N Engl J Med $361: 40-51$ 